\title{
El esfuerzo laboral, la autoestima y la salud mental en mujeres trabajadoras de Lima Metropolitana
}

\author{
The work effort, self-esteem and mental health in working women in \\ Metropolitan Lima \\ Alejandro E. Loli P., Fernando Arias G., Javier del Carpio G.,Jesús Arce V. Melisa Reyes I., Nery Anyaco P. \\ Universidad Nacional Mayor de San Marcos. Lima, Perú \\ (RECIBIDO 05/01/2014, AcEPTADO 24/03/2014)

\section{RESUMEN} \\ Muchas personas e investigadores han manifestado su preocupación por la salud mental y \\ física de las mujeres que trabajan fuera del hogar. Se argumenta que la diversidad de roles \\ y las horas dedicadas al trabajo acarrean daños. Al respecto existen múltiples pesquisas, \\ pero los investigadores encuentran aquello que están buscando. Así, pocos artículos se han \\ publicado respecto a los factores protectores; por tanto, el objetivo del presente capítulo es \\ mirar hacia los dos lados. Se administró una encuesta a 292 mujeres peruanas a fin de ob- \\ servar las correlaciones entre el desequilibrio, el esfuerzo y la recompensa; la salud física y \\ la mental; la autoestima, la autoeficacia, el distrés y la integración familiar. Se conformaron \\ cinco grupos: solo amas de casa, amas de casa con algún ingreso extra (cuidado de niños, \\ lavado, planchado, venta de artículos entre familiares y amistades, etc.), amas de casa y \\ profesionales, empleadas sin responsabilidades hogareñas y profesionales sin responsabilidad \\ de hogar. En general, el último grupo exhibió mejores puntuaciones en las variables incluidas, \\ mientras las mujeres dedicadas solo al hogar presentaron las menores. Una conclusión: no \\ necesariamente los múltiples roles significan salud física y mental deteriorada. Se sugieren \\ investigaciones adicionales.
}

Palabras clave: esfuerzo laboral, autoestima, salud física y mental, mujeres, Lima, Perú

\begin{abstract}
Many people and researchers have expressed concern about the mental and physical health of women working outside the home, it is argued that the diversity of roles and the hours spent at work entail damage. In this regard there are many researches, but the researchers found what they are looking for. Thus, few articles have been published regarding protective factors; therefore, the objective of this chapter is to look at the two sides. A survey was administered to 292 Peruvian women to observe the correlations between the imbalance, effort and reward, the physical and mental health, self -esteem, self-efficacy, distress, and family integration. Consisted of five groups: only housewives, housewives some extra income
\end{abstract}


(babysitting, washing, ironing, selling items among family and friends, etc.), Housewives and professionals, employed no household responsibilities, and home professionals without liability. In general, the latter group showed better scores on the variables included, while only dedicated women had the lowest scores. One conclusion: the multiple roles do not necessarily mean physical and mental health deteriorated. Additional research is suggested.

Keywords: Labor effort, self-esteem, physical and mental health, women, Lima , Peru.

\section{INTRODUCCIÓN}

Según el IX Censo Nacional de Población de 2007, en Perú el $33.72 \%$ de la mujeres de 15 a 29 años estaba ocupada; de quienes contaban entre 30 y 44 años, laboraba el $47.80 \%$; en tanto el $39.56 \%$ de entre 45 a 64 años lo hacía y el $13.66 \%$ estaba ocupada entre quienes contaban con más de 65 años. Dedicadas al cuidado del hogar, sin trabajar, los porcentajes fueron: entre 15 y 29 años, $36.13 \%$; entre 30 y 44 años, $47.82 \%$; entre 45 y 64 años, $54.38 \%$ y más de 65 años, 59.68\% (Instituto Nacional de Estadística e Informática, 2013). Así, pues, se denota la importancia de las personas de sexo femenino en la economía y en la sociedad peruana. Por tanto, es imprescindible conocer su sentir respecto a sus trascendentes labores y papeles.

Ahora bien, el concepto de rol o papel se refiere al conjunto de obligaciones, expectativas y derechos adjudicados por la sociedad a los individuos en razón de su posición en ella (Gelles y Levine,2000). Por tanto, el papel proporciona a las personas un marco de referencia para dar significado, propósito y maneras de actuar en su existencia (Reitzes y Mutran, 1994). Así, de manera tradicional, se ha dividido el trabajo entre hombres y personas del sexo femenino. A los primeros se les ha adjudicado el papel de proveedores del hogar, mientras las segundas deben encargarse de las labores domésticas: limpieza, preparación de alimentos, atención a los niños, lavado, planchado, cuidado de otras personas, etc. Por ende, la incursión de las mujeres en el campo del trabajo remunerado implica, además, la adjudicación de tareas adicionales, lo cual conlleva mayor tiempo dedicado al trabajo (tanto retribuido como en el hogar). Al respecto, en México, Oliveira, Ariza y Eternod (1996) estimaron en 9.3 horas diarias el trabajo de las mujeres por encima del de los hombres, pero las mujeres casadas y con baja escolaridad dedicaron 14.3 horas al día. Las repercusiones pueden reflejarse en mayor distrés.

El distrés, por su parte, presenta consecuencias en la salud física y mental si alcanza niveles elevados. Existen muchas evidencias sobre la aseveración anterior. Por ejemplo, Segerstrom y Miller (2004) analizaron las investigaciones publicadas en los últimos 30 años (hasta esa fecha) respecto a la relación entre el estrés crónico y el sistema inmunológico. La conclusión: a mayor distrés, menor defensa presenta el organismo, lo cual da sustento a enfermedades infecciosas e, inclusive, probabilidad de contraer cáncer. 
La Organización Mundial de la Salud indicó en su informe de 2001 (p-4): “Sabemos hoy que la mayor parte de las enfermedades, tanto psíquicas como orgánicas, están influidas por una combinación de factores biológicos, psicológicos y sociales".

No solo ha existido preocupación sobre la mujer peruana respecto a su estado físico y mental con relación al trabajo. Desde años atrás se han presentado inquietudes al respecto en el ámbito mundial, especialmente por la incursión cada vez más acentuada de las mujeres en el campo laboral.

Por ejemplo, Murphy (2003) describió los problemas de salud de las mujeres en razón de la inequidad de sexo, colocando atención a los diferentes riesgos a los cuales están expuestas las mujeres: problemas ginecológicos, morbilidad y mortalidad maternal, falta de planificación familiar, aborto, VIH/SIDA, violencia y abuso sexual. Todo lo anterior fue ligado por la autora a aspectos psicológicos y sociales.

El punto de vista anterior se refuerza con investigaciones provenientes de diversos países. Así, por caso, Sánchez-López, Aparicio-García y Dresch (2006) analizaron los estados anímicos y de salud de 500 personas en España, elegidas al azar. Las mujeres denotaron salud y autoestima menor al compararlas con los varones, así como mayor ansiedad. Sin embargo, en cuanto a la satisfacción con la vida no hubo diferencias. No obstante, al considerar una submuestra de personas empleadas y desempleadas, desaparecieron las diferencias en la salud percibida (aunque no en cuanto a las visitas al médico, siendo las mujeres quienes informaron de mayor número) y la autoestima; en cambio, los hombres dieron una media mayor en referencia a la satisfacción vital. Las autoras analizaron esas variables entre las amas de casa, las empleadas y quienes carecían de empleo. Las primeras dieron las medias siguientes: dolencias físicas, 17.10; salud física percibida, 6.83; ansiedad total, 29.49; autoestima, 21.12; las puntuaciones presentadas por las desempleadas: dolencias físicas, 16.31; salud física percibida, 7.13; ansiedad total, 25.11 y autoestima, 20.72; las empleadas: dolencias físicas, 16.36; salud física percibida, 7.37; ansiedad total, 25.11 y autoestima, 20.72. Merecen destacarse las puntuaciones de la satisfacción con la vida: amas de casa, 7.70; desempleadas, 6.69 y empleadas, 7.49. Pueden verse, entonces, las repercusiones de la situación laboral, aunque las puntuaciones no resultaron drásticamente diferentes.

Matud y Bethencourt (2000) encontraron como variables predictoras de la depresión (medida mediante el Cuestionario de Salud General de Goldberg ), entre 180 amas de casa en las Islas Canarias, la autoestima, la satisfacción con el papel como ama de casa y el apoyo social (con signo negativo); en cambio, la emocionalidad ante el estrés resultó con signo positivo. Estos resultados van de acuerdo a lo esperado por los planteamientos teóricos.

Uno de los modelos con mucha difusión en los últimos años es el de desequilibrio entre esfuerzo y recompensa (Siegrist, 1996; Siegrist, 2010; Siegrist, Starke, Chandola, Godin, Marmot, Niedhammer y Peter, 2004), el cual postula repercusiones negativas en la salud psicológica y física cuando se presenta una inequidad entre la energía puesta en las acciones de trabajo y la retribución esperada; cuando no se compensan, cuando hay desequilibrio continuado, surge el distrés, el cual 
conlleva afecciones a la salud física, especialmente en cuanto a las enfermedades cardiovasculares. El modelo incluye, de hecho, cuatro componentes: esfuerzo físico, esfuerzo mental o sobreimplicación, recompensas y desequilibrio entre los elementos previos. Por estas razones se incluyó en esta investigación un cuestionario tendiente a evaluar las percepciones de las mujeres en estas variables.

Ahora bien, mucho se ha investigado respecto a los aspectos negativos de los diversos papeles asumidos por las mujeres y sus repercusiones negativas en la salud física y mental, incluyendo el distrés. Empero, parece no haberse puesto tanta atención a los factores paliativos o amortiguadores tales como la autoestima, la autoeficacia y la integración familiar.

La autoestima se conceptúa como los sentimientos de agrado y amor hacia una(o) misma(o); por ende, la baja autoestima se caracteriza por el desagrado (Brown, Dutton y Cook, 2001). En casos extremos existe odio y se cae, entonces, en situaciones patológicas (Baumeister, Tice y Hutton, 1989, citados en Brown, Dutton y Cook, 2001).

Se han documentado las relaciones entre la autoestima y la salud. Por ejemplo, en España, Matud (2004) efectuó una investigación en la cual aplicó una encuesta a 2,383 mujeres, de 18 a 65 años; sus hallazgos: a mayor autoestima, mejor salud y menores síntomas depresivos, así como menor ansiedad y disfunción social; igualmente, su estilo de afrontamiento era más racional y menos emocional. Aunque los aspectos demográficos mostraron poca influencia, se presentó una ligera tendencia entre las mujeres con estudios universitarios, las casadas, quienes tenían uno o dos hijos y quienes ocupaban puestos de mayor importancia.

Como ya se mencionó, uno de los problemas señalados por muchos autores es la multiplicidad de roles jugados por las mujeres con un trabajo remunerado. Empero, Ruderman, Ohlott, Panzer y King (2002), empleando métodos cualitativos, encontraron, al investigar a mujeres en puestos de gerencia, que los papeles desempeñados en su vida personal contribuían con mayores beneficios psicológicos, consejo y apoyo emocional, práctica en tareas múltiples, oportunidad para enriquecer sus habilidades interpersonales y liderazgo, todo lo cual incrementaba su efectividad gerencial. Desde el ángulo cuantitativo, la diversidad de roles se relacionó con la satisfacción en la vida, la autoestima, la autoaceptación, así como a las habilidades gerenciales. Como puede verse fácilmente, estos resultados van en contra de la suposición de la disminución de la salud psicológica al tener múltiples responsabilidades.

En relación con la autoeficacia, definida como la creencia de tener el conjunto de capacidades para organizar y llevar a cabo las acciones necesarias para llegar a ciertos logros (Bandura, 2000), puede pensarse en una relación negativa con el distrés pues, según el punto de vista de Lazarus y Folkman $(1984,1987)$, este surge cuando la persona, ante una situación amenazadora, siente que no cuenta con los elementos suficientes para hacerle frente.

En cuanto al núcleo familiar, este puede constituir una fuente de apoyo emocional. Por ende, puede pensarse en una asociación favorable con la salud física y mental. 
Hasta la culminación de este estudio, eran escasas las investigaciones de relevancia científica respecto a las mujeres en el Perú y, de manera particular, sobre las variables analizadas en el presente estudio. Sin duda, los argumentos generales y aquellos estudios propios de los países latinoamericanos servirán de guía y fundamento para analizar los resultados, debido a la similitud histórica en la evolución del rol de la mujer, cada vez más dinámica y compleja y, por tanto, sujeta a posibles variaciones en la salud física y mental.

Con base en todo lo anterior, se establecieron las siguientes hipótesis nulas:

- No habrá diferencia entre las variables esfuerzo físico, esfuerzo mental (sobreimplicación), recompensa, desequilibrio, salud física, salud mental, autoestima, autoeficacia, integración familiar y distrés, en razón de los papeles jugados por las mujeres de la muestra.

- Las correlaciones entre dichas variables no serán significativas. Las hipótesis alternas van en el sentido contrario a las expresadas.

\section{MÉTODO}

\section{Muestra}

La muestra, de conveniencia, estuvo formada por 292 mujeres habitantes de Lima metropolitana, Perú, a quienes se invitó a responder voluntariamente a los instrumentos mencionados párrafos adelante.

Las edades se distribuyeron así: $2.7 \%$ declaró entre 18 y 20 años, el $78.5 \%$ estuvo entre los 21 y los 50 años y el 18\% en edades superiores. El 8\% dejó en blanco este rubro.

El $33.1 \%$ vivía sin pareja, mientras el $66.6 \%$ habitaba con pareja; el resto no contestó. Con respecto a la escolaridad, el $4.8 \%$ contaba con educación primaria; el $25.3 \%$, con secundaria; el $11.6 \%$, con grado de bachiller; el $25.9 \%$. con 3 años o más de profesional; el $24.9 \%$, con profesional completa y el $6.1 \%$, con posgrado. No respondió a esta pregunta el $0.3 \%$

Del total de la muestra, el $17.7 \%$ se declaró solo ama de casa mientras el $16 \%$, aparte de esta ocupación, tenía ingresos adicionales por vender productos entre vecinas, familiares y conocidas, planchar, cuidar niños de otras mujeres, etc. El $8.9 \%$ eran microempresarias y amas de casa; el $4.4 \%$, además de ser ama de casa, era también empleada manual; el 8.9\%, ama de casa y oficinista; el 30.4\%, profesional y ama de casa; el $0.3 \%$ declaró ser solo microempresaria; el $0.7 \%$ era empleada manual sin ser ama de casa; el 8.9\% solo se clasificó como profesional sin ser ama de casa y el $2.7 \%$ era oficinista sin responsabilidades hogareñas.

Dado el bajo porcentaje en algunas categorías respectivas a la ocupación, se recodificaron estas, quedando de la siguiente forma: solo ama de casa, ama de casa con ingresos adicionales, profesional y ama de casa, empleada sin ser ama de casa y profesional sin ser ama de casa. 


\section{Instrumentos}

Esfuerzo-recompensa. Este cuestionario fue desarrollado por Siegrist (1996, Siegrist et al., 2004). Contiene 23 reactivos, los cuales miden tres variables principales: esfuerzo intrínseco (responsabilidades y presiones), recompensas y esfuerzo mental (sobreimplicación). Las dos primeras variables mencionadas se responden en una escala de cinco opciones, mientras la última contiene cuatro. Con ellas se obtiene una escala secundaria: desequilibrio entre los esfuerzos y las recompensas. Originalmente el instrumento fue diseñado para muestras de trabajadores, por tanto, fue necesario transformar ligeramente la redacción de los ítems con la finalidad de ajustarlo a las amas de casa; por ejemplo, en vez de "En mi trabajo tengo mucha responsabilidad" se cambió a "En mi hogar tengo mucha responsabilidad". Macías Robles, Fernández-López, Hernández-Mejía, Cueto-Espinar, Rancaño y Siegrist (2003) llevaron a cabo la validación y la confiabilización del instrumento original entre trabajadores de la salud en España.

WHOQOL-Bref. Este inventario fue desarrollado por la Organización Mundial de la Salud [World Health Organization] (1997). Bonicato y Soria (1998) lo adaptaron a la lengua castellana. Mide las variables: salud física, salud mental, relaciones sociales y medio ambiente. Para los efectos de la presente investigación, en consideración al marco teórico, solo se incluyeron las dos primeras variables. Las respuestas deben proporcionarse en una escala de cinco opciones.

Autoestima. Reidl (1981), psicóloga mexicana, diseñó este instrumento, el cual consta de 20 reactivos con tres opciones.

Autoeficacia. Sus posibilidades de respuesta son cuatro; consta de ocho enunciados. (Juárez García, 2005).

Distrés. Cuatro enunciados del cuestionario de Cohen, Karmarck y Mermelstein (1983), adaptado a México por González Ramírez y Landero Hernández (2007). Contiene una escala de cinco opciones.

Integración familiar. Fue construido por Olson y Wilson (1982). La versión castellana fue utilizada por Ramírez Serrano (2007) en México; consta de 11 enunciados y cinco opciones.

Es importante poner énfasis en un aspecto: al ofrecer diversas opciones de respuesta en los instrumentos, el error del método común se ve reducido, de acuerdo a las recomendaciones de Podsakoff, MacKenzie, Podsakoff \& Lee (2003).

\section{Procedimiento}

Los instrumentos se aplicaron en los hogares o en el lugar de trabajo, según donde se encontrara la participante. 


\section{Análisis de la información}

Los datos se procesaron con el SPSS 21, haciendo uso de las medidas de tendencia central, a fin de conocer la intensidad del comportamiento de la muestra en cada una de las variables, la correlación de Pearson para establecer las relaciones entre las variables y el Anova para conocer el grado de significancia en la relación de las variables con los diversos grupos de la muestra.

\section{RESULTADOS}

Las medias aritméticas y las desviaciones estándar de las variables incluidas en la presente investigación se incluyen en la tabla 1.

La interpretación de las primeras cuatro variables es un tanto más problemática, pues aunque las respuestas pueden darse en cinco opciones en cuanto al esfuerzo físico y las recompensas, respecto al esfuerzo mental el cuestionario contiene cuatro posibilidades. Además, la sumatoria no se divide entre el número de reactivos y la fórmula de desequilibrio es un tanto más compleja. Aquí solo puede mencionarse la indicación de Juárez García (2011), en el sentido de que la media latinoamericana es de 13 en la variable esfuerzo físico, por ende, los resultados de esta muestra se localizan muy cercanamente a esa cifra.

La media latinoamericana para recompensa es de 43 (Juárez García, 2011), en tanto que el promedio de la muestra presente (47.54) se sitúa unas cuantas unidades por encima. El promedio internacional para desequilibrio es 0.65 , mientras las participantes en esta investigación promediaron 0.17; es decir, el desequilibrio experimentado por las mujeres participantes en esta investigación resultó bajo en relación con el promedio de otros países. En cuanto al sobreinvolucramiento o esfuerzo mental la media internacional es de 14, casi dos unidades menos que en esta muestra,11.90. No obstante, las puntuaciones obtenidas en esta investigación confirman la semejanza de la muestra con los resultados internacionales.

Una nota adicional: las distribuciones de frecuencias de tres de estas variables resultaron asimétricas (en contra de las correspondientes a las demás variables), la de recompensa estuvo muy cargada hacia la derecha (o sea, la mayoría de las mujeres se sintieron muy premiadas por sus labores), mientras las correspondientes al desequilibrio y al esfuerzo físico, especialmente la primera, se inclinaron hacia la izquierda, indicando menor intensidad de estos factores. .

Para facilitar la interpretación de las puntuaciones obtenidas en esta muestra, en las demás escalas la suma de cada persona se dividió entre el número de reactivos integrantes de cada escala. En todos los casos las puntuaciones van de menor a mayor; es decir, entre más elevada sea la puntuación, la persona se siente más en posesión del fenómeno medido.

Las opciones de la salud, tanto física como mental, son cinco; así las medias de 3.37 y 3.38, respectivamente, se localizaron solo unas cuantas centésimas del punto medio de la escala. 
La escala de autoestima se responde con tres opciones. La media es de 2.40 , lo cual indica una elevada sensación de amor de las participantes hacia ellas mismas.

La autoeficacia, con una puntuación de 3.14 implicó una percepción cercana al máximo: 4.

Las participantes denotaron percibir cercanía mediana dentro del clan familiar pues, de cinco opciones, el promedio resultó 3.61, o sea, casi en el punto medio de la escala.

La media aritmética del distrés (2.97) se situó apenas unas centésimas por debajo del punto medio de la escala, la cual proporciona cinco opciones.

Tabla 1. Medias aritméticas y desviaciones estándar de las variables bajo análisis. Muestra de Lima, Perú

\begin{tabular}{lccc}
\hline & Media & Desviación típica & Opciones de la escala \\
\hline ESFUERZO FÍSICO & 13.37 & 5.494 & 1 a 5 \\
RECOMPENSA & 47.54 & 8.462 & 1 a 5 \\
DESEQUILIBRIO & .17 & .142 & -- \\
ESFUERZO MENTAL & 11.90 & 3.049 & 1 a 4 \\
SALUD FÍSICA & 3.37 & .598 & 1 a 5 \\
SALUD MENTAL & 3.38 & .567 & 1 a 5 \\
AUTOESTIMA & 2.40 & .329 & 1 a 3 \\
AUTOEFICACIA & 3.14 & .476 & 1 a 5 \\
\hline INTEGRACIÓN FAMILIAR & 3.61 & .662 & 1 a 5 \\
DISTRÉS & 2.97 & .690 & 1 a 5 \\
\hline
\end{tabular}

$\mathrm{n}=292$

Nota: Las puntuaciones de las primeras cuatro variables se presentan de acuerdo a las indicaciones de Siegrist et al. (2004), mientras las seis últimas están dadas, para su mejor interpretación, en las opciones indicadas.

En la tabla 3 se incluyen las correlaciones Pearson entre las diversas variables. Solo una asociación no resultó estadísticamente significativa: entre la autoeficacia y el esfuerzo mental o sobreimplicación.

La correlación más alta $(-.805, \mathrm{p} \leq .01)$ apareció entre el desequilibrio y la recompensa, lo cual no es nada sobresaliente pues en el cálculo de la primera variable mencionada se considera, precisamente, la segunda. Por ende, existe cierta redundancia al respecto, algo semejante sucedió con la primera variable y el esfuerzo extrínseco $(.783, \mathrm{p} \leq .01)$.

$\mathrm{Al}$ analizar las correlaciones de las variables pertenecientes al modelo de esfuerzorecompensa (insertas en las cuatro primeras columnas de la tabla 2), se constata la adecuación entre dicho modelo de acuerdo a las expectativas teóricas y las demás variables medidas. 
Así, el esfuerzo físico presentó correlaciones negativas con las variables ajenas al modelo, excepto con el distrés, en cuyo caso fue positiva. En cambio, la recompensa mostró asociaciones positivas con las mencionadas variables; empero, con el distrés fue negativa. Algo semejante ocurrió con el esfuerzo mental (sobreimplicación) y con el desequilibrio.

La asociación más elevada, fuera de las mencionadas, se presentó entre las vertientes de la salud: física y mental $(.699, \mathrm{p} \leq .01)$, seguida por la salud mental (.530) y la integración familiar.

Las variables salud física, salud mental, autoestima, autoeficacia e integración familiar resultaron con correlaciones positivas entre ellas, mientras fueron negativas con el distrés.

Así, pues, puede constatarse la adecuación de los resultados con los aspectos teóricos.

Con referencia a las confiabilidades de los instrumentos, se obtuvieron los siguientes índices alfa. Como se puede apreciar, razonablemente aceptable para estos fines, dándole estabilidad y confiabilidad.

Tabla 2. Índice de confiabilidad para cada una de las variables

\begin{tabular}{lc}
\hline Variables & Confiabilidad \\
\hline Esfuerzo extrínseco (físico) & 0.836 \\
Recompensa & 0.885 \\
Esfuerzo intrínseco o sobreimplicación (mental) & 0.613 \\
Salud física & 0.744 \\
Salud mental & 0.753 \\
Autoestima & 0.789 \\
Autoeficacia & 0.852 \\
Integración familiar & 0.898 \\
Distrés & 0.718 \\
\hline
\end{tabular}

Cronbach's Alpha

Como se puede deducir en la tabla 2, todos los índices se catalogan como aceptables de acuerdo a los estándares internacionales. Las cifras correspondientes se incluyeron también en la diagonal principal de la tabla 3. La variable desequilibrio se calcula a partir de las puntuaciones de esfuerzo y recompensa y un factor de corrección; por tanto, no puede calcularse el índice de confiablidad. Empero, a partir de las altas confiabilidades de las dos variables mencionadas, puede deducirse también la adecuación del desequilibrio al modelo de confiabilidad. 
EL ESFUERZO LABORAL, LA AUTOESTIMA Y LA SALUD MENTAL EN MUJERES TRABAJADORAS DE LIMA METROPOLITANA

Tabla 3. Correlaciones Pearson entre las variables.

Mujeres de Lima, Perú, $\mathrm{n}=292$

\begin{tabular}{|c|c|c|c|c|c|c|c|c|c|c|}
\hline VARIABLES & 1 & 2 & 3 & 4 & 5 & 6 & 7 & 8 & 9 & 10 \\
\hline 1. ESFUERZO FÍSICO & .836 & & & & & & & & & \\
\hline 2. RECOMPENSA & $-.604 * *$ & .885 & & & & & & & & \\
\hline 3. DESEQUILIBRIO & $.783 * *$ & $-.805 * *$ & -- & & & & & & & \\
\hline 4. ESFUERZO MENTAL & $.469 * *$ & $-.340 * *$ & $.374^{* *}$ & .613 & & & & & & \\
\hline 5. SALUD FÍSICA & $-.330 * *$ & $.426^{* *}$ & $-.359^{* *}$ & $-.389^{* *}$ & .744 & & & & & \\
\hline 6. SALUD MENTAL & $-.292 * *$ & $.361 * *$ & $-.256 * *$ & $-.333 * *$ & $.699 * *$ & .753 & & & & \\
\hline 7. AUTOESTIMA & $-.357 * *$ & $.410 * *$ & $-.324 * *$ & $-.239 * *$ & $.398 * *$ & $.466^{* *}$ & .789 & & & \\
\hline 8. AUTOEFICACIA & $-.118^{*}$ & $.192 * *$ & $-.134 *$ & -.056 & $.301 * *$ & $.317^{* *}$ & $.344 * *$ & .852 & & \\
\hline 9. INTEGRACIÓN FAMILIA & $-.265^{* *}$ & $.413 * *$ & $-.268 * *$ & $-.274 * *$ & $.489 * *$ & $.530^{* *}$ & $.469 * *$ & $.397 * *$ & .898 & \\
\hline 10. DISTRÉS & $.308^{* *}$ & $-.299 * *$ & $.223^{* *}$ & $.339 * *$ & $-.381 * *$ & $-.471 * *$ & $-.325 * *$ & $-.148 *$ & $-.416 * *$ & .718 \\
\hline
\end{tabular}

$* \mathrm{p} \leq .05$

$* * \mathrm{p} \leq .01$

Nota: se incluyeron los índices de confiabilidad alfa en la diagonal principal.

En la tabla 4 pueden verse las medias aritméticas, las desviaciones estándar y la significación estadística del modelo de esfuerzo-recompensa (Siegrist, 1996, 2010) clasificadas de acuerdo a las ocupaciones de las mujeres participantes. Ahí puede constatarse la puntuación menor obtenida por quienes eran profesionales sin tener responsabilidades hogareñas en las variables esfuerzo extrínseco o físico, desequilibrio y esfuerzo intrínseco o mental o sobreimplicación. En cambio, su puntaje fue mayor en cuanto a la variable recompensa, aunque en esta variable las diferencias solo se aproximaron a la significación estadística $(\mathrm{p} \leq .05)$ pues esta resultó de .07. Al efectuar la prueba post-hoc de Dunette (en la cual no se asumen varianzas iguales entre los grupos) en todas las variables mencionadas las diferencias fueron significativas entre las amas de casa y las profesionales sin responsabilidades hogareñas. De la misma manera, las profesionales y amas de casa resultaron con mejor puntuación con relación a las amas de casa con ingresos adicionales.

Tabla 4. Anova de variables del modelo de esfuerzo-recompensa por ocupación. Mujeres de Lima, Perú. $\mathrm{n}=292$

\begin{tabular}{clcccc}
\hline & & $\mathrm{n}$ & Media & $\begin{array}{c}\text { Desviación } \\
\text { estándar }\end{array}$ & Significación: \\
\hline & Solo ama de casa & 52 & 14.4808 & 5.65855 & \\
& Ama de casa con ingresos adicionales & 112 & 14.8036 & 5.28441 & $\mathrm{p} \leq .001$ \\
ESFUERZO & Profesional y ama de casa & 89 & 12.4607 & 5.32374 & \\
FÍSICO & Empleada sin ser ama de casa & 11 & 12.3636 & 5.02539 & \\
& Profesional sin ser ama de casa & 26 & 8.6538 & 3.55463 & \\
\hline
\end{tabular}


Alejandro E. Loli P., Fernando Arias G., Javier Del Carpio G.,Jesús Arce V. Melisa Reyes I., Nery Anyaco P.

\begin{tabular}{clcccc}
\hline & Solo ama de casa & 52 & 45.9808 & 9.42772 & \\
& Ama de casa con ingresos adicionales & 112 & 46.5000 & 8.24840 & \\
RECOMPENSA & Profesional y ama de casa & 89 & 49.2135 & 8.41734 & $\mathrm{p}=.077$ \\
& Empleada sin ser ama de casa & 11 & 46.5455 & 10.26025 & \\
& Profesional sin ser ama de casa & 26 & 49.4615 & 5.65849 & \\
\hline \multirow{2}{*}{ DESEQUILIBRIO } & Solo ama de casa & 52 & .2065 & .21007 & \\
& Ama de casa con ingresos adicionales & 112 & .1937 & .13971 & $\mathrm{p}=.007$ \\
& Profesional y ama de casa & 89 & .1531 & .10520 & \\
& Empleadas sin ser ama de casa & 11 & .1676 & .11794 & \\
& Profesional sin ser ama de casa & 26 & .0994 & .05247 & \\
\hline \multirow{2}{*}{ ESFUERZO } & Solo ama de casa & 52 & 12.3654 & 2.99692 & \\
& Ama de casa con ingresos adicionales & 112 & 12.6786 & 2.95679 & $\mathrm{p} \leq .001$ \\
& Profesional y ama de casa & 89 & 11.3371 & 3.02243 & \\
& Empleada sin ser ama de casa & 11 & 11.5455 & 2.87623 & \\
& Profesional sin ser ama de casa & 26 & 10.0000 & 2.52982 & \\
\hline
\end{tabular}

Al aplicar el análisis de la varianza de un factor a las variables mencionadas en la tabla 5, se encontraron diferencias significativas en todas las variables consideradas. Las mejores puntuaciones se presentaron en el caso de las profesionales sin responsabilidades hogareñas, con excepción de la variable autoeficacia, en la cual las mujeres profesionales y, al mismo tiempo, amas de casa rindieron la mejor puntuación.

Cuando se aplicó la prueba post-hoc de Dunnette, las diferencias importantes se presentaron entre las amas de casa y profesionales y las profesionales sin responsabilidades hogareñas y las demás categorías, indicando la importancia de la educación para disfrutar de la vida, por un lado, y, por el otro, para enfrentar con mayor éxito las vicisitudes de la existencia cotidiana.

Tabla 5. Anova de las variables indicadas por ocupación.

Mujeres de Lima, Perú. n= 292

\begin{tabular}{clcccc}
\hline & VARIABLE & $\mathrm{n}$ & Media & $\begin{array}{c}\text { Desviación } \\
\text { típica }\end{array}$ & Significación \\
\hline \multirow{5}{*}{ SALUD FÍSICA } & Solo ama de casa & 52 & 3.2143 & .63036 & \\
& Ama de casa con ingresos adicionales & 112 & 3.2487 & .55403 & \\
& Profesional y ama de casa & 89 & 3.5425 & .53243 & $\mathrm{p}=.001$ \\
& Empleada sin ser ama de casa & 11 & 3.5325 & .73980 & \\
& Profesional sin ser ama de casa & 26 & 3.5549 & .69321 & \\
\hline \multirow{5}{*}{ SALUD } & Solo ama de casa & 52 & 3.2280 & .55528 & \\
MENTAL & Ama de casa con ingresos adicionales & 112 & 3.2538 & .58249 & \\
& Profesional y ama de casa & 89 & 3.5425 & .46947 & $\mathrm{p} \leq .001$ \\
& Empleada sin ser ama de casa & 11 & 3.2727 & .70986 & \\
& Profesional sin ser ama de casa & 26 & 3.7912 & .46814 & \\
\hline
\end{tabular}


EL ESFUERZO LABORAL, LA AUTOESTIMA Y LA SALUD MENTAL EN MUJERES TRABAJADORAS DE LIMA METROPOLITANA

\begin{tabular}{clcccc}
\hline & Solo ama de casa & 52 & 2.2548 & .31874 & \\
& Ama de casa con ingresos adicionales & 112 & 2.3192 & .30486 & \\
AUTOESTIMA & Profesional y ama de casa & 89 & 2.5421 & .29183 & $\mathrm{p} \leq .001$ \\
& Empleada sin ser ama de casa & 11 & 2.3364 & .30667 & \\
& Profesional sin ser ama de casa & 26 & 2.6250 & .31281 & \\
& Solo ama de casa & 51 & 3.0417 & .48713 & \\
& Ama de casa con ingresos adicionales & 112 & 3.0536 & .45727 & \\
AUTOEFICACIA & Profesional y ama de casa & 89 & 3.2823 & .46064 & $\mathrm{p}=.003$ \\
& Empleada sin ser ama de casa & 11 & 3.1023 & .49943 & \\
& Profesional sin ser ama de casa & 26 & 3.2740 & .45692 & \\
\hline \multirow{5}{*}{ INTEGRACIÓN } & Solo ama de casa & 52 & 3.5017 & .65617 & \\
& Ama de casa con ingresos adicionales & 112 & 3.4667 & .65105 & \\
& Profesional y ama de casa & 89 & 3.7681 & .63222 & $\mathrm{p}=.005$ \\
& Empleada sin ser ama de casa & 11 & 3.6281 & .90950 & \\
& Profesional sin ser ama de casa & 26 & 3.8217 & .52663 & \\
\hline \multirow{2}{*}{ DISTRÉS } & Solo ama de casa & 52 & 3.1859 & .75093 & \\
& Ama de casa con ingresos adicionales & 112 & 3.0833 & .71939 & \\
& Profesional y ama de casa & 88 & 2.8182 & .58046 & $\mathrm{p} \leq .001$ \\
& Empleada sin ser ama de casa & 11 & 2.8485 & .68902 & \\
& Profesional sin ser ama de casa & 26 & 2.6026 & .53349 & \\
\hline
\end{tabular}

Con referencia a las demás variables, los resultados pueden verse en la tabla 4 . Ahí se constata la mejor puntuación obtenida por las profesionales sin ser amas de casa. En cambio, cuando las mujeres eran solo amas de casa, la tendencia fue en el sentido de presentar menores puntajes. Cuando se analizaron las pruebas post-hoc efectuadas con la $\mathrm{C}$ de Dunette, las diferencias mayores se encontraron entre las amas de casa solamente y las profesionales, ya sea que fueran amas de casa o no.

\section{DISCUSIÓN}

Los resultados aquí incluidos dan sustento al modelo de esfuerzo-recompensa de Siegrist $(1996,2004)$ en una muestra de mujeres peruanas. Por tanto, este capítulo constituye una aportación al conocimiento respectivo. Las hipótesis fueron confirmadas. Así, pues, se agrega un indicio más a los ya existentes sobre la validez del modelo.

Otras investigaciones apoyan los hallazgos del presente trabajo. Por ejemplo, Feldman, Vivas, Lugli, , Zaragoza y Gómez (2008) investigaron a 402 trabajadores de Caracas, Venezuela, con edades comprendidas entre 27 y 71 años. Encontraron asociaciones positivas entre las gratificaciones de la relación trabajo y familia con la autoestima y el bienestar, y asociaciones negativas con la depresión, la ansiedad y la sintomatología física. En cambio, las interferencias entre esas dos esferas acarrearon menor salud física y mental.

Digna de mención es la investigación llevada a cabo por Escalera-Izquierdo y Sebatián-Herranz (2000), quienes encontraron una tendencia de las mujeres con empleo remunerado a reportar salud incrementada al investigar a 340 mujeres de 
Guadalajara (España); si bien proporcionaron más altas puntuaciones en salud y vitalidad al compararlas con las amas de casa, sus puntajes fueron menores en padecimientos crónicos. Con referencia al bienestar, las diferencias entre los dos grupos no fueron significativas. Las variables con mayor contribución a la salud psicológica fueron: la autoestima, el ajuste marital, el ajuste maternal y los ingresos entre las empleadas; en cambio, las condiciones de trabajo tanto en casa como en el empleo y el ajuste maternal mostraron signos negativos. Las variables con mayor peso fueron: el nivel educativo y la autoestima; con sentido negativo: conductas de riesgo y la tensión de roles, con respecto a la salud física. En contra de la opinión general, el número de horas de trabajo no marcaron influencia ni en la salud psicológica ni en la física en ambos grupos. Estos hallazgos contradicen la idea de mayores problemas de las mujeres con empleo remunerado al compararlas con las amas de casa.

De la misma manera, Norton, Gupta, Stephens, Martire y Townsend (2005) encontraron entre 195 mujeres, quienes ejercían los papeles de madre, cuidadora de algún progenitor, esposa y empleada, una asociación negativa entre el dominio percibido de las tareas (equivalente a la autoeficacia) y el incremento en el cuidado de las personas mencionadas. Las recompensas incrementaron dicha autoeficacia en los cuatro roles referidos, mientras el estrés la disminuyó en cuanto al papel como empleada. No obstante, los cambios en la autoeficacia no decrementaron la importancia asignada por ellas a sus papeles. Se concluye, así, la permanencia de la trascendencia asignada por las mujeres a sus roles. Esta investigación confirma el hallazgo de la presente: las recompensas se asociaron a la autoeficacia.

A mayor abundamiento, ya desde 1986 Baruch y Barnett informaron de sus hallazgos al investigar, entre 283 mujeres norteamericanas, las relaciones entre los papeles desempeñados (empleada, esposa y madre) y el bienestar (autoestima, felicidad, optimismo y satisfacción) por un lado, y por el otro, depresión. Aplicaron el análisis de regresión a fin de controlar los efectos de la edad, el ingreso económico y el nivel educativo, encontrando aspectos importantes: los roles por sí mismos no mostraron relación con el bienestar, excepto en el caso de las mujeres con trabajo remunerado, quienes exhibieron mayor autoestima. La conclusión de estas autoras: no es tan importante el número de papeles desempeñados, sino su calidad; igualmente no debería hablarse de bienestar o malestar en general, sino de cada una de las facetas de estos constructos.

A conclusión semejante arribó Noor (2004), al investigar a 147 mujeres inglesas: la importancia adjudicada al trabajo exacerbó el sentimiento de este como un obstáculo para la convivencia familiar y, como consecuencia, una influencia negativa hacia el bienestar, quizá debido al valor asignado al núcleo familiar.

Respecto a la salud física, Ferrer-Pérez, Bosch-Fiol y Gili-Planas (1998), en una investigación entre 540 mujeres de las Islas Baleares, encontraron mejores niveles de salud en las mujeres con trabajo remunerado fuera del hogar, en comparación con las amas de casa, pese a la mayor frecuencia entre estas de consumo de alcohol y de tabaco. 
Otra área con posibilidades de arrojar más luz respecto a las relaciones entre el trabajo y la salud es la personalidad. Para el caso, Noor (2003) efectuó una encuesta entre 187 mujeres empleadas en Oxford, Inglaterra. Encontró el peso de variables de personalidad (neuroticismo y extraversión) sobre el bienestar de las participantes, especialmente en lo tocante a la interferencia de la familia sobre el trabajo

Generalmente se piensa en una relación entre el número de horas dedicadas a las labores hogareñas y el distrés, especialmente en las féminas con un trabajo remunerado. Esta es una situación demandante de investigación, inclusive, dadas las situaciones actuales donde, pese a que el "machismo" todavía permea grandes sectores de la población, los hombres participan cada vez más en las labores del hogar. En este punto, Noor (1995) encontró entre 153 mujeres casadas inglesas una mayor repercusión en su bienestar de las horas dedicadas por el marido a las labores hogareñas, en vez de las horas empleadas por ellas, lo cual parece indicar la importancia de la integración familiar. Sería necesario, en investigaciones futuras, analizar la relación del tiempo dedicado por las empleadas con responsabilidades de hogar y el apoyo brindado no solo por el marido sino también por los hijos y otros parientes.

Este punto se vio reforzado por una investigación efectuada por Noor (1995) entre 115 mujeres empleadas de tiempo completo. El apoyo familiar exhibió correlaciones positivas con la felicidad y negativas con la salud. Como era de esperarse, la correlación entre estas dos últimas variables fue positiva.

Así pues, con base en los resultados de la presente investigación, así como de las otras citadas, se arrojan serias dudas sobre la idea del deterioro físico y mental en las mujeres con un empleo remunerado fuera del hogar; al contrario, este hecho parece incrementar su salud, tanto mental como física. El hecho de adquirir varios papeles y el número de horas dedicadas a ellos per se parece no incidir negativamente en las otras esferas de su vida; parece más importante la calidad de las relaciones intra y extrafamiliares, así como la importancia adjudicada a los diversos roles.

No obstante, es necesario indicar las limitaciones de la presente investigación. Se trató de una muestra de conveniencia en vez de una aleatoria; además, los datos se obtuvieron en una sola ciudad y con un número reducido de instrumentos. Por ende, los resultados no pueden generalizarse a otras latitudes.

En el futuro nuevas variables deben ser investigadas, pues pueden significar repercusiones en el bienestar de las mujeres casadas y/o amas de casa. Por ejemplo, el número de hijos y las edades de ellos. Probablemente, por ejemplo, los niños pequeños requieren mayor atención, $\mathrm{y}$, por ende, más tiempo, lo cual puede incrementar el distrés.

Otro punto importante para investigaciones futuras es el relativo a la importancia adjudicada por las mujeres a sus roles. Surgen, por lo pronto, varias preguntas: ¿a cuál papel otorgan mayor valor, al de empleada o al de ama de casa?, ¿cómo se relaciona esa importancia con los posibles conflictos entre trabajo y familia?, ¿existen relaciones entre esas trascendencias adjudicadas y la salud física y mental, la felicidad y el distrés? 


\section{CONCLUSIONES}

1. En una evaluación general, las mujeres de Lima - Perú obtienen un puntaje promedio, ligeramente alto, en relación con lo que ocurre en otros países respecto a esfuerzo físico y recompensa, disminuyendo su puntuación respecto a desequilibrio y esfuerzo mental. Entre tanto, respecto a salud física y salud mental se mantienen en el nivel medio, mejorando en autoestima y autoeficacia. Mientras que en integración familiar tienen un puntaje medio, en distrés se sitúan en un nivel bajo. Entre ellas, las profesionales sin responsabilidad del hogar tuvieron mejores puntuaciones y las mujeres dedicadas solo al hogar presentaron menor puntuación. Lo que significa que no necesariamente los múltiples roles implican salud física y mental deteriorada.

2. Existe asociación significativa entre las variables consideradas (esfuerzo físico, recompensa, desequilibrio, esfuerzo mental, salud física, salud mental, autoestima, autoeficacia, integración familiar y distrés) en la muestra total, excepto entre esfuerzo mental y autoeficacia, donde la relación no es significativa. Esto lleva a concluir que existe consistencia y confiabilidad de los instrumentos.

3. Existen diferencias significativas respecto del esfuerzo físico y esfuerzo mental entre las participantes de la muestra, siendo mayor el puntaje obtenido por las amas de casa con ingresos adicionales y menor la puntuación de los profesionales sin ser amas de casa. Mientras que respecto del desequilibrio, el puntaje más alto lo obtienen las que se dedican solo a actividades de amas de casa y el más bajo las profesionales sin ser amas de casa; entre tanto, respecto de la recompensa, el puntaje mayor le corresponde a los profesionales sin ser amas de casa y el puntaje menor a solo amas de casa. En otros términos, la muestra agrupada, en virtud de las actividades que realizan, permiten establecer diferencias respecto del esfuerzo físico, esfuerzo mental, recompensa y desequilibrio, denotando diferencias en las reacciones de las personas, en función de las actividades a las que se dedican frente a las diversas variables.

4. Existen diferencias significativas respecto de la salud física, salud mental, autoestima, autoeficacia, integración familiar y distrés en toda la muestra agrupada por ocupaciones, obteniendo una mayor puntuación el grupo de profesionales sin responsabilidad hogareña, con excepción respecto de la autoeficacia, donde los puntajes fueron mejores en el grupo de profesionales con responsabilidades de ama de casa, indicando la importancia de la educación para disfrutar de la vida y enfrentar con mayor éxito las vicisitudes cotidianas.

\section{REFERENCIAS BIBLIOGRÁFICAS}

Escalera-Izquierdo, M. E. y Sebatián-Herranz, J. (2000). Trabajo y salud en la mujer: análisis comparativo de mujeres con trabajo remunerado y amas de casa. Clínica y Salud, 11(2), 195-229. 
Ferrer-Pérez, V. A. Bosch-Fiol, E. y Gili-Planas, M. (1998). Aspectos diferenciales en salud entre las mujeres que trabajan fuera del hogar y las amas de casa. Psicothema,.10(1), 53-63.

Feldman, L., Vivas, E., Lugli, Z., Zaragoza, J. \& Gómez V. (2008). Relaciones trabajofamilia y salud en mujeres empleadas. Salud Pública de México. 50(6), 482-489.

Gelles, R J. y Levine, A. (2000). Sociología con aplicaciones en países de habla hispana. $6^{\mathrm{a}}$. Ed. México: McGraw-Hill.

Instituto Nacional de Estadística e Informática (2013). Censos Nacionales 2007: XI de Población y VI de Vivienda. Recuperado el 16 de julio de http//: www.inei.gob.pe.

Matud, M. P. \& Bethencourt, J. M. (2000). Ansiedad, depresión y síntomas psicosomáticos en una muestra de amas de casa. Revista Latinoamericana de Psicología, 32(1), 91-106.

Matud, M. P. (2004). Autoestima en la mujer: un análisis de su relevancia en la salud. Avances en Psicología Latinoamericana, 22, 129-140.

Murphy, E. M. (2003). Being Born Female Is Dangerous for Your Health. American Psychologist, 58(3), 205-210.

Noor, N.M. (1995). Role quality and womens' psychological well-being: locus of control and support as moderators. Journal of Community \& Applied Social Psychology,5, 259-272.

Noor, N. M. (1997). The relationship between wives' estimates of time spent doing housework, support and wives' well-being. Journal of Community \& Applied Social Psychology, 7, 413-423.

Noor, N. M. (2003). Work- and family-related variables, work-family conflict and women's well-being: some observations. Community, Work \& Family, 6(3), 297-319.

Noor, N. M. (2004). Work-Family Conflict, Work- and Family-Role Salience, and Women's Well-Being. The Journal of Social Psychology, 2004, 144(4), 389-405.

Norton, T. Gupta, A. Stephens, M. Martire, L. \& Townsend, A. (2005). Stress, rewards, and change in the centrality of women's family and work roles: mastery as a mediator. Sex Roles 52(5/6), 325-335.

Oliveira, O., Ariza, M. y Eternod, M. (1996). Trabajo e inequidad de género. En Oliveira, O. (coord.). La condición femenina: una propuesta de indicadores. México: Sociedad Mexicana de Demografía/Consejo Nacional de Población.

Organización Mundial de la Salud, WHO. (1997). Measuring quality of life. The WHOQOL-100 AND WHOBREF. Autor: Ginebra.

Organización Mundial de la Salud (2001). Informe de la salud en el mundo. Salud mental: nuevos conocimientos. Nuevas esperanzas. Ginebra: Autor.

Podsakoff, P., MacKenzie, S., Podsakoff, N \& Lee, J. (2003). Common method biases in behavioral research: A critical review of the literature and recommended remedies. Journal of Applied Psychology, 88, 879-903. 
Reitzes, D. C., \& Mutran, E. J. (1994). Multiple roles and identities: Factors Influencing self-esteem among middle-aged working men and women. Social Psychology Quarterly, 57, 313-325.

Ruderman, M. N., Ohlott, P. J., Panzer, K. \& King, S. N. (2002). Benefits of multiple roles for managerial women. Academy of Management Journal, 45(2), 369-386.

Sánchez-López, M.P., Aparicio-García, M.E. \& Dresch, V. (2006). Ansiedad, autoestima y satisfacción autopercibida como predictores de la salud: diferencias entre hombres y mujeres. Psicothema, 18(3), 584-590.

Segerstrom, S.C. \& Miller, G. E. (2004). Psychological stress and the human immune system: A meta-analytic study of 30 years of inquiry. Psychological Bulletin, 130(4), 601-630.

Siegrist, J. (1996). Adverse health effects of high-effort/low-rewards conditions. Journal of occupational health psychology. 1, 27-41.

Siegrist, J. (2010). Effort- reward imbalance at work and cardiovascular diseases. International Journal of Occupational Medicine and Environmental Health, 23(3), $279-285$.

Siegrist, J. Starke, D., Chandola, T., Godin, I., Marmot, M., Niedhammer, I. \& Peter, R. (2004). The measurement of effort-reward imbalance at work: European comparisons. Social science and medicine, 58, 1483-1499. 